\title{
Small ELM regimes with good confinement on JET and comparison to those on ASDEX Upgrade, Alcator C-mod, and JT-60U
}

J. Stober ${ }^{1}$, P. Lomas ${ }^{2}$, G. Saibene ${ }^{3}$, Y. Andrew ${ }^{2}$, P. Belo ${ }^{4}$, G.D. Conway ${ }^{1}$,L.D. Horton ${ }^{1}$, M. Kempenaars ${ }^{5}$, H.-R. Koslowski ${ }^{6}$, A. Loarte ${ }^{3}$, G.P. Maddison ${ }^{2}$, M. Maraschek ${ }^{1}$, D.C. McDonald ${ }^{2}$, A.G. Meigs ${ }^{2}$, P. Monier-Garbet ${ }^{7}$, D.A. Mossessian ${ }^{8}$, M.F.F. Nave ${ }^{4}$, N. Oyama ${ }^{9}$, V. Parail ${ }^{2}$, Ch.P. Perez ${ }^{6}$, F. Rimini ${ }^{7}$, R. Sartori ${ }^{3}$, A.C.C. Sips ${ }^{1}$, P.R. Thomas ${ }^{7}$, contributors to the EFDA-JET work programme, and the ASDEX Upgrade Team

1 Max-Planck-Institut für Plasmaphysik, EURATOM-Association, D-85748 Garching, Germany

2 Euratom/UKAEA Association, Culham Science Centre, Abingdon, OX14 3DB, UK

${ }^{3}$ EFDA Close Support Unit (Garching), 2 Boltzmannstrasse, Garching, Germany

${ }^{4}$ Associação EURATOM/IST, Centro de Fusão Nuclear, 1049-001 Lisbon, Portugal

${ }^{5}$ FOM-Rijnhuizen, Association Euratom-FOM, TEC, PO Box 1207, Nieuwegein, The Netherlands

${ }^{6}$ Forschungszentrum Jülich GmbH, Institut fur Plasmaphysik, EURATOM Association, Trilateral Euregio Cluster, 52425 Jülich, Germany

7 Association Euratom-CEA, Cadarache, F-13108 St. Paul-lez-Durance, France

${ }^{8}$ Massachusetts Institute of Technology Cambridge, MA, USA

9 JAERI, Naka Fusion Research Establishment, Naka-machi, Naka-gun, Ibaraki-ken, 311-01, Japan

e-mail: Joerg.Stober@ipp.mpg.de

\begin{abstract}
Since it is uncertain if ITER operation is compatible with type-I ELMs, the study of alternative H-mode pedestals is an urgent issue. This paper reports on experiments on JET aiming to find scenarios with small ELMs and good confinement, such as the type-II ELMs in ASDEX Upgrade, the enhanced Dalpha H-mode in Alcator C-mod or the grassy ELMs in JT-60U. The study includes shape variations, especially the closeness to a double-null configuration, variations of $q_{95}$, density and beta poloidal. Hmode pedestals without type-I ELMs have been observed only at the lowest currents ( $\leq 1.2 \mathrm{MA}$ ), showing similarities to the observations in the devices mentioned above. These are discussed in detail on the basis of edge fluctuation analysis. For higher currents, only the mixed type-I/II scenario is observed. Although the increased inter-ELM transport reduces the type-I ELM frequency, a single type-I ELM is not significantly reduced in size. Obviously, these results do question the accessibility of such small ELM scenarios on ITER, except perhaps the high beta-poloidal scenario at higher $q_{95}$, which could not be tested at higher currents at JET due to limitations in heating power.
\end{abstract}

\section{Introduction}

Estimates of the ELM amplitude for ITER indicate that the type-I ELMs for the standard Q=10 scenario at $q_{95}=3$, could be so large that the divertor lifetime would be as low as a few hundred discharges, although this depends strongly on the physics assumptions [1]. To be prepared for the worst case, techniques for ELM mitigation/control are being developed. This contribution reports on efforts to establish in JET regimes with smaller (or no) ELMs, but nevertheless good H-mode energy confinement and sufficient particle exhaust, testing strategies derived from successful experiments on other devices, i.e. type-II ELMs on ASDEX Upgrade, grassy ELMs on JT-60U, and the "Enhanced D-Alpha"(EDA) H-Mode on Alcator C-mod. The Quiescent $\mathrm{H}$-mode $(\mathrm{QH})$ on DIII-D and ASDEX Upgrade has also been attempted in JET and is reported in a separate contribution [2].

$\mathrm{H}$-modes with increased edge transport in between type-I ELMs have been observed in JET [3] in high-triangularity (high- $\delta$ ) equilibria at high density ( $>80 \%$ of the Greenwald density 
$\left.n_{G W}\right)$ and $q_{95}=3 \ldots 3.5$, up to a plasma current of 3 MA [4]. Due to some similarities in the $D_{\alpha}$ traces and due to the observed increase of MHD-activity and power flux over the separatrix between ELMs these phases were called mixed type-I/II phases in analogy to similar observations on other machines. In JET, the type-II phases in between typeI ELMs do usually not show highfrequent irregular bursty events as are found on other devices. Only at the lowest plasma currents, i.e. $I_{p}=$ 1.1 MA, smaller bursts in between the bigger ones could be found in
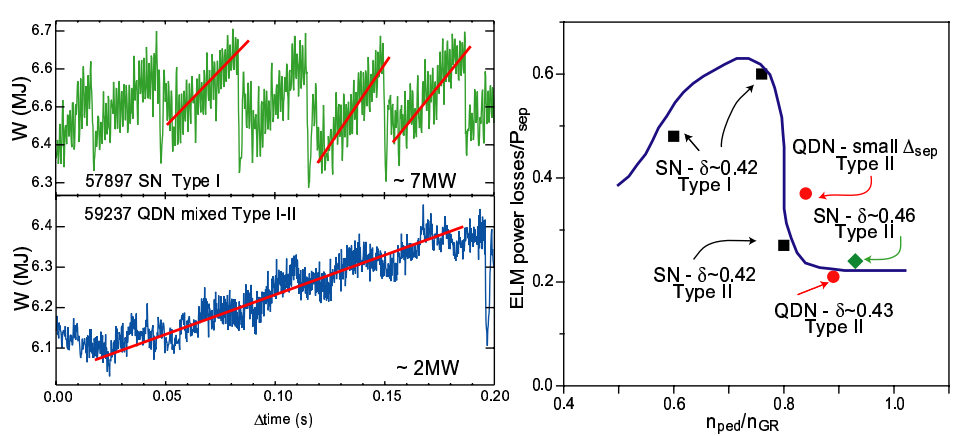

Figure 1: Mixed type-I/II H-modes in JET, $I_{p}=2.5$ MA. Time evolution of the stored energy (left) and ELM contribution to the average power flux across the separatrix $\left(P_{\text {heat }}-P_{\text {rad,bulk }}\right)$. The blue curve sketches the behaviour for the most frequently used HT3-configuration ( $q_{95}=3.5$, black squares), to compare to the behaviour of other configurations ("ITER-like": green, $Q D N$ : red, both $q_{95}=3.0$ ) magnetic and edge-density signals.

Fig. 1 shows that during these mixed type-I/II phases the inter-ELM power flux can double from $40 \%$ (as observed in pure type-I ELM phases at slightly lower density) to $80 \%$ of the time-averaged power crossing the separatrix. Unfortunately the remaining $20 \%$ is delivered by type-I ELMs similar in size to those observed in the pure type-I ELM phases at densities below $0.8 n_{G W}$. The enhanced inter-ELM transport is related to an increase in strength of broadband MHD modes moving in the direction of the electron diamagnetic drift with frequencies between $10 \mathrm{kHz}$ and $40 \mathrm{kHz}$ [5], which are called at JET washboard-modes (WB) [6]. In the same frequency range one finds enhanced density fluctuations close to the pedestal top with the FIR-interferometer [7].

In the following sections recent efforts to further increase the inter-ELM transport on JET are described. As these were stimulated by successful scenarios on other devices this paper focuses on the inter-machine comparision of the results. Additional information, omitted due to space limitations, can be found in [7] (JET experiments) and [8] (inter-machine comparison).

\section{Effect of DN and increased $q_{95}$ on the JET mixed type-I/II ELM regime}

The results of the ASDEX Upgrade type-II ELMs showed that a quasi-DN (QDN) configuration is an essential ingredient for that regime [9]. Therefore a SN-configuration of JET which shows the mixed type-I/II regime was modified to be close to DN and still compatible with operation at 2.5 MA plasma current. Unfortunately, as Fig. 1 shows, the ratio of inter-ELM losses to typeI ELM losses could not be significantly changed. Both for SN and QDN configurations, $q_{95}$ has been increased, following the experience for EDA-H-mode on Alcator C-mod and ASDEX Upgrade $\left(q_{95}>3.5\right)$ as well as on JT-60U $\left(q_{95} \approx 4 \ldots 6\right)$. An increase of $q_{95}$ from 3 to 4 , or, in a different configuration, from 3.6 to 4.7 led to a significant decrease (20\%) of the pedestal density for the same level of gas puff, combined with an increased ELM frequency. Starting from an anchor point at $I_{p}=2.5 \mathrm{MA}$ and $B_{t}=2.7 \mathrm{~T}$, similar results have been obtained either by raising the magnetic field or by lowering the plasma current. With increased gas puff the density can be recovered, but only at reduced energy confinement and very high frequent ELMs; probably type-III. This means that a regime with $\mathrm{H} 98 \approx 1$ and $q_{95}>4$ has not been achieved at JET with any ELM type at plasma currents above 2 MA. There appears to be a significant dependence of particle confinement on $q_{95}$, specifically between ELMs [7], but the reasons for this are still unclear. 


\section{Dimensionless identity with small ELM regimes on Alcator C-mod and ASDEX Up- grade}

A possible way to analyse the failure to obtain pure small ELM phases in JET at plasma currents above $2 \mathrm{MA}$ are dimensionless identity experiments at low plasma current matching the respective plasmas in smaller machines. This requires a match of $\rho^{*}, v^{*}, \beta, q_{95}$, and $\varepsilon$ as well as a scaled match of the poloidal cross section. Under such conditions dimensional plasma parameters should follow well defined size scalings if not dominated by atomic physics or effects on the scale of the Debye length. Since the H-mode pedestal region is most affected by ELMs, the above mentioned dimensionless parameters should be matched in this region for comparison of the ELM behaviour. A source of ambiguity is usually the additional heating since NBI heating clearly involves atomic physics and scaled ICRH frequencies may not be available. Also fueling by wall recycling and gas puff does not happen according to the scaling requirement (i.e. the radial width of the particle-source profile does not scale with the major radius). Earlier pedestal dimensionless identity experiments showed that for example the $\mathrm{L} / \mathrm{H}$ threshold [10] in JET and ASDEX Upgrade can be well scaled using the same value of $\rho^{*}, v^{*}, \beta$, but not between ASDEX Upgrade and Alcator C-mod [11] hindering similar EDA-dimensionless-identity experiments such as those reported below for JET and Alcator C-mod.

\subsection{Pedestal dimensionless identity to EDA-H-mode}

The EDA-H-mode in Alcator C-mod is found above certain thresholds in $\delta, q_{95}$, and $n_{p e d}$ and shows good H-mode confinement close to the (non-steady-state) ELM-free H-mode [12]. It is characterized by continous MHD activity and density fluctuations between $60 \mathrm{kHz}$ and $120 \mathrm{kHz}$ of rather narrow band width located in the steep gradient region. These fluctuations are called the quasi-coherent mode (QCM). At higher input power a transition to a grassy ELM regime is observed and the edge MHD frequency drops and becomes broadband (see for example fig. 4 in [12], where the grassy ELM MHD is observed in the whole frequency range between the original QCM and zero). After some iterations a very good shape match between JET and Alcator C-mod has been achieved. The reference Alcator C-mod discharge shows the usual EDA behaviour. The experiments are described in detail in $[13,14]$. The main difficulty in JET is the diagnosis of the edge pedestal as the JET diagnostic set was not designed to operate in this operational range $\left(I_{p}=0.65 \mathrm{MA}, B_{t}=0.9 \mathrm{~T}\right)$. An uncertainty on the pedestal pressure of about 50\% remained, spanning the scaled value from Alcator $\mathrm{C}$-mod. In the steep gradient zone at the plasma edge good agreement of $\nabla n_{e}, \nabla T_{e}$ with the scaled values of Alcator C-mod was observed. Nevertheless it remains uncertain to what extent dimensionless identical plasmas have been achieved. Additionally, the plasma behaviour depends crucially on small variations of the lower triangularity and on the heating method, which was either NBI or 2nd harmonic hydrogen minority heating with ICRH, in contrast to 1st harmonic minority heating at Alcator C-mod. No controlled EDA modes have been found at JET, but phases with a steady pedestal were observed for several $\tau_{E}$ (up to $1 \mathrm{~s}$ ). Magnetic and density fluctuation patterns observed with pick-up coils and a reflectometer are variable in terms of strength and bandwidth. Often the fluctuations are enhanced in the $10 \mathrm{kHz}$ to $20 \mathrm{kHz}$ range and rotate in the direction of the electron diamagnetic drift as well. Occasionally the bandwith $\Delta f$ is lower than $1 \mathrm{kHz}$. In such cases at least two harmonics of a base frequency of 10 to $15 \mathrm{kHz}$ are observed (higher ones are very weak). According to the dimensionless identity scaling this frequency does correspond to the frequency range of 60 to $90 \mathrm{kHz}$ at Alcator C-mod, which is within the frequency rance of the quasi coherent mode. Nevertheless, multiple harmonics are never observed at Alcator C-mod so that the observations on both machines do not exactly match. In JET these phases without ELMs and with constant $n_{\text {ped }}, T_{\text {ped }}$ are observed with NBI as well as with ICR heating With ICR fluctuation patterns with a band-width 
compareable to the QCM have been observed [13,14]). A pure NBI-heated case is shown in fig. 2, which shows one of the longest phases with steady pedestal conditions. Under these conditions edge density fluctuations are strongest in the upper half of the steep gradient zone, whereas in Alcator C-mod they are found in the lower
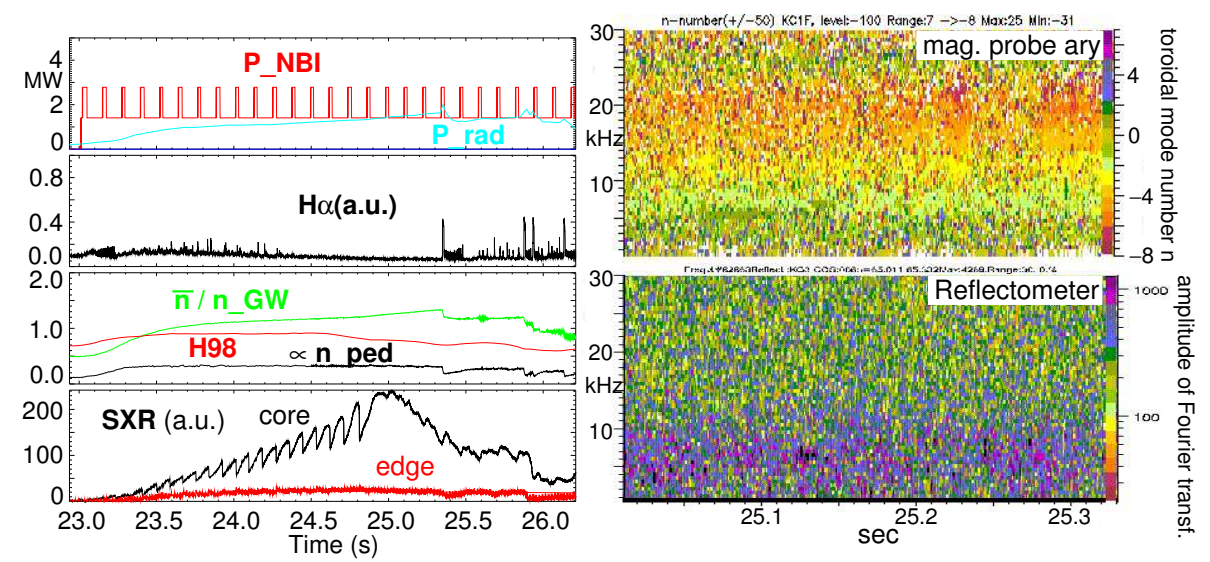

Figure 2: JET pulse 62663, $I_{p}-0.68 M A, B_{t}=0.94 T, q_{95}=4.2$, aimed to be dimensionless identical to Alcator C-mod plasmas. The figures to the right show the toroidal mode number analysis (neg. numbers correspond to the electron diamagnetic drift direcrion) and density fluctuations measured in the steep gradient region with the reflectometer $\left(34 \mathrm{GHz} \hat{=} 1.4 \cdot 10^{19} \mathrm{~m}^{-3}\right)$

half. With ICRH as

well as with NBI, an increase of radiation leads to a transition to type-III ELMs with poorer confinement. Interestingly, the increase of radiation is observed simultaneosly with a central peaking of the density profile. This recalls observations in DIII-D [15], ASDEX Upgrade [16], Alcator C-mod [17], and JET [18], in which a slow central density peaking has been observed for broad or off-axis peaked profiles of auxillary heating. Without further analysis it remains a speculation that similar effects could play a role in this case. So far, we simply note that in any case the NBI heating profile in JET is much broader than the profile of 1 st harmonic ICR minority heating in Alcator C-mod. This could also be true for the 2 nd harmonic ICR minority heating in JET, which has a poorer single pass absorption and possibly additional impurity resonances at the plasma edge. Thus the available auxillary heating schemes do violate the dimensionless scaling approach. It remains to be verified that this is really the reason for the observed differences.

\subsection{Dimensionless identity approach for type-II ELMs as in ASDEX Upgrade}

In [9] three criteria were introduced for the characterization of type-II ELMs in ASDEX Upgrade: good H-mode confinement, quasi continous heat flux to divertor as measured by the infrared cameras (IR) and broadband fluctuations in density and magnetics in the steep gradient zone of the edge pedestal ranging from $20 \mathrm{kHz}$ to $40 \mathrm{kHz}$. Type-II ELMs are observed in QDN configurations with $q_{95}>3.5 \ldots 4.0$ in a narrow density band just below the transition to type-III ELMs. As described in [9], a reduction of $q_{95}$ below the threshold leads to a mixture with type-I ELMs and the bandwidth of the inter-ELM fluctuations is reduced. Under these conditions the fluctuation occur in quite regular bursts $(\approx 0.5 \ldots 1 \mathrm{kHz})$. Such a phase has been revisited for the comparisons in this paper. The result is shown in fig. 3. The highly localised reflectometer measurement shows only during the mixed I/II phase two bands around 13 and 25 $\mathrm{kHz}$ whereas the MHD is more broadband in between 10 and $30 \mathrm{kHz}$. This MHD structure is sharp enough to determine its mode number, yielding $n \leq 4$ and $m \geq 12$ again in the direction of the electron diamagnetic drift. The MHD fluctuations between 150 and $200 \mathrm{kHz}$ had been overlooked earlier, but they are present in all type-II phases published in [9] and disappear at transitions to type-III ELMs. The mode numbers are in this case $n=5 \ldots 7, m \geq 12$, electron direction. More recent type-II experiments show that the MHD/reflectometer footprint are not found at higher power (as for example high $\beta$ improved H-modes [19]) and/or strong ICRH 
(> $3 \mathrm{MW}$ ) although the other two criteria still hold. Due to differences in the sets of poloidal-field coils a close shape match of the high- $\delta$ ASDEX Upgrade equilibria was impossible at JET, so that a QDN configuration with the same average $\delta$ has been chosen. To reduce the diagnostic problems at very low currents discussed in the previous section, the plasma current in JET $\left(I_{p}=0.9 \mathrm{MA}, B_{t}=1.2 \mathrm{~T}\right)$ was chosen to correspond to a 1 MA ASDEX Upgrade discharge although only 0.8 MA were run at ASDEX Upgrade with the high$\delta$ shape used in [9]. Fig. 4 shows that indeed long phases without ELMs and steady pedestal parameters have been achieved. As in the case of the EDA identity (fig. 2) the central density slowly rises, terminated by the loss of sawteeth and a radiation collapse. As discussed above, this can be due to the unmatched heat deposition profile. In this case also the mismatch in upper triangularity may be responsible for the behaviour, since it is found on ASDEX Upgrade, that high upper triangularity favours the occurrence of slow density peaking. Fig. 4 shows the spectrogram of an reflectometer channel in the steep gradient region and the MHD mode number analysis. Two sharp bands are observed with the reflectometer at approximately 8 and $15 \mathrm{kHz}$, rather close to what is expected by scaling the ASDEX Upgrade results (see fig. 3, scaling factor $\left.\left(a_{J E T} / a_{A U G}\right)^{1.25} \approx 2.0\right)$. As in ASDEX Upgrade the MHD fluctuations are less sharp and again they move in the direction of the electron drift. The n-number of the mode between 15 and $20 \mathrm{kHz}$ is close to 4 , the minimum m-number is estimated to be 7 , a lower limit depending on the present set of pick-up coils. These numbers also relate well to the findings on ASDEX Upgrade $(n=4, m>12)$. After $23.7 \mathrm{sec}$ the bandwidth of the modes measured with the reflectometer increases and the frequency drops in contrast to the MHD anal-

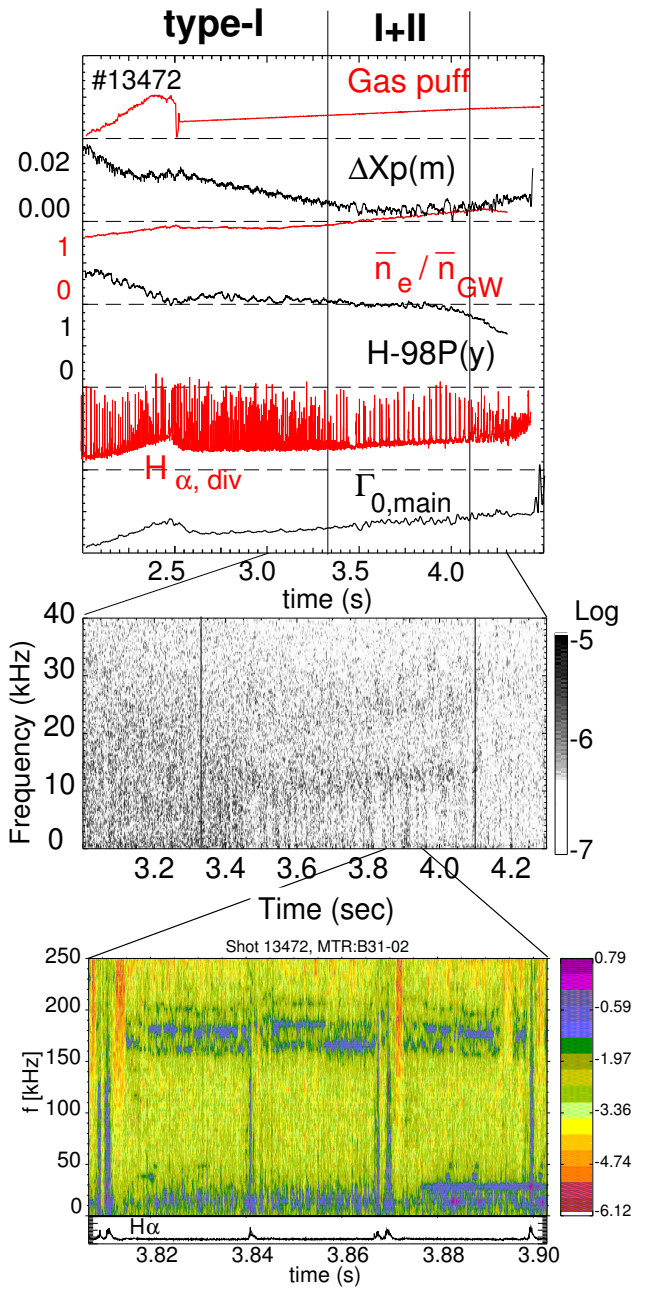

Figure 3: ASDEX Upgrade pulse 13472 0.8 MA, 2.0 T, $q_{95}=3.9$, (see also [9]), corresponding to a mixed type-I/I phase. Middle: density fluctuation as seen by a reflectometer channel in the steep gradient region close to the pedestal top. Bottom: spectrogram of a magnetic signal together with an $H_{\alpha}$ trace from the outer divertor. ysis. Due to the strong radial localisation of the reflectometer this could be due to the still slightly increasing edge density, shifting the location of the measurement further towards the separatrix and possibly away from the mode. In any case this example shows that bandwidth and frequency of the observation are subject to a significant variation. We also note that the upper frequency bands observed in ASDEX Upgrade (see fig. 3) should appear around $100 \mathrm{kHz}$ in JET according to the scaling. Indeed a weak band is observed in the magnetics at $110 \mathrm{kHz}$, rotating in the right direction, with $n \approx 2$ which is lower than in ASDEX Upgrade. After having successfully established the scenario at JET using the dimensionless identity approach, the intension was to increase plasma current and magnetic field. The plasma current was increased from 0.9 MA to 1.2 MA and 1.5 MA. For 1.2 MA only mixed type-I/II phases were obtained with the type-II phases lasting up to $0.3 \mathrm{sec}$. At $1.5 \mathrm{MA}$ only type-I ELMy H-modes were found. Although the search at the higher currents was rather coarse due to time limitations, the failure indicates that it is more difficult if not impossible to get the 
pure type-II phases with increasing current, especially since the low current scenario was found in three campaigns during 2003 in a quite straight forward manner, using a coarse gas scan.

4. The high $\beta_{\text {pol }}$ grassy ELM scheme of JT-60U applied to JET and ASDEX Upgrade Another small ELM regime with good confinement are the grassy ELMs observed in JT-60U. The most recent publication is [20]. As reported by JT-60U [21] a high value of $\beta_{p o l}$ facilitates operation with grassy ELMs most probably due to to the stabilising effect of a strong Shafranovshift $[20,22]$. The regime is observed as $\beta_{\text {pol }}$ exceeds a value of $1.4 \ldots 2.0$ depending on $q_{95}$ and $\delta$. High values of the latter two quantities favour the occurrence of the grassy ELM regime. Typical values are $\beta_{p o l} \geq 1.6$ and $q_{95} \approx 6$ for $\delta \approx 0.5$. Usually the regime is operated with an internal transport barrier using early heating to avoid the occurrence of sawteeth. The grassy ELM regime has significantly lower colisionality $\mathrm{v}_{e e}^{*} \approx 0.1$ as compared to the high density small ELM regimes discussed above which have $2 \geq v_{e e}^{*} \geq 0.5$. This triggered experiments to transfer it to JET and ASDEX Upgrade. These experiments were not meant to match the JT60U plasmas in the strict sense of a dimensionless identity experiment, but the key ingredients high $\beta_{\text {pol }}$, high $\delta$ and high $q_{95}$ have been used for plasmas that showed already signs of mixed type-I/II ELMs at lower values of $q_{95}$ and $\beta_{p o l}$.

\section{1. high $\beta_{p o l}$ grassy ELM experiments at JET}

In JET, the shape developed for the identity experiment with ASDEX Upgrade was also used for the high $\beta_{\text {pol }}$ experiment, with somewhat increased plasma current (1.2 ..1.5 MA), but strongly increased magnetic field (2.7 T) and plasma heating (17 MW to $23 \mathrm{MW}$, i.e. all power available on the day). This also means that the configuration was close to DN. Above a value of 1.8 only very small ELMs are observed [7]. The energy carried by them is so small that it cannot be quantified in contrast to the type-I ELMs at lower $\beta_{p o l}$ which each carry more than $5 \%$ of the stored energy. The magnetic spectrogram and the fast $D_{\alpha}$ trace of such a JET-grassyELM phase are shown in fig. 5. They are compared to a grassy ELM phase of JT-60U discharge E42857, with $\beta_{\text {pol }} \approx 2.0$. The spectrograms look similar. In particular there are no modes seen in the range of a few $10 \mathrm{kHz}$ or at even higher frequencies. Such high values of $\beta_{\text {pol }}$ could only be achieved at JET at $I_{p}=1.2 \mathrm{MA}$ due to limitations in heating power. There is no evidence that this is a physics based limit. In parallel to these experiments another set of high $\beta_{p o l}$ discharges have been run at JET to study the effect of the high $\beta_{p o l}$ on the core transport together with a reversed $q$ - profile and consequently lower $l_{i}$ and higher edge currents, using Lower Hybrid pre-heating and early main heating. Further differences are that the plasma shape was clearly a lower SN shape and that the pedestal collisionality was about 50\% lower. Interestingly no grassy ELMs were observed even at $\beta_{p o l}=1.9$ [7]. Obviously the
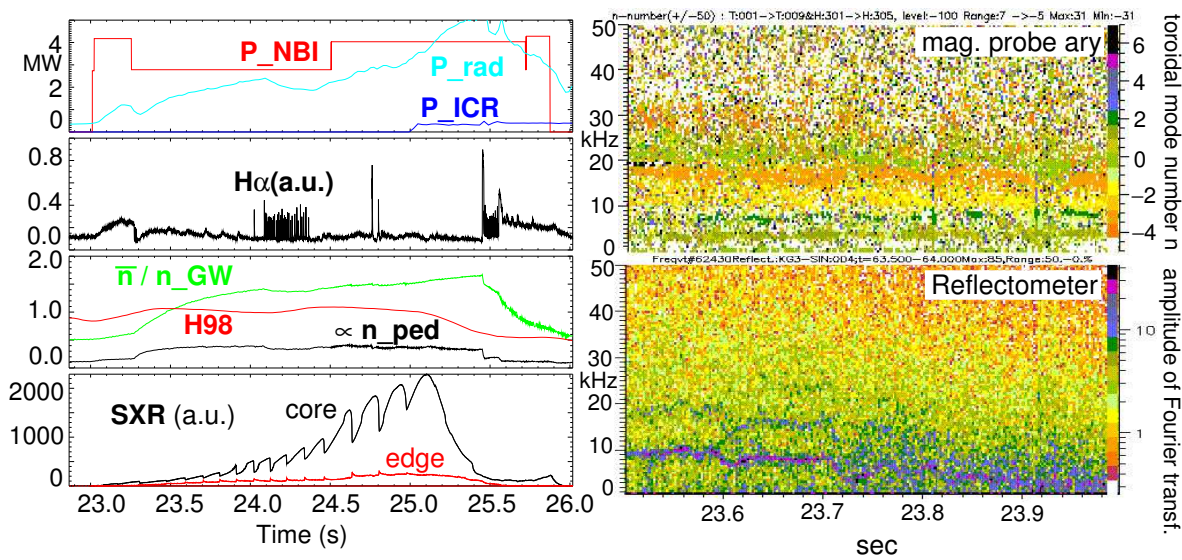

Figure 4: JET pulse 62430, $I_{p}-0.86 M A, B_{t}=1.15$ T, $q_{95}=4.1$, aimed to be dimensionlessly identical to ASDEX Upgrade type-II H-modes. The figures to the right show the toroidal mode number analysis (neg. numbers correspond to the electron diamagnetic drift direcrion) and density fluctuations measured

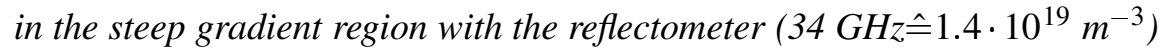


influence of the above mentioned differences has to be separated by further experiments.
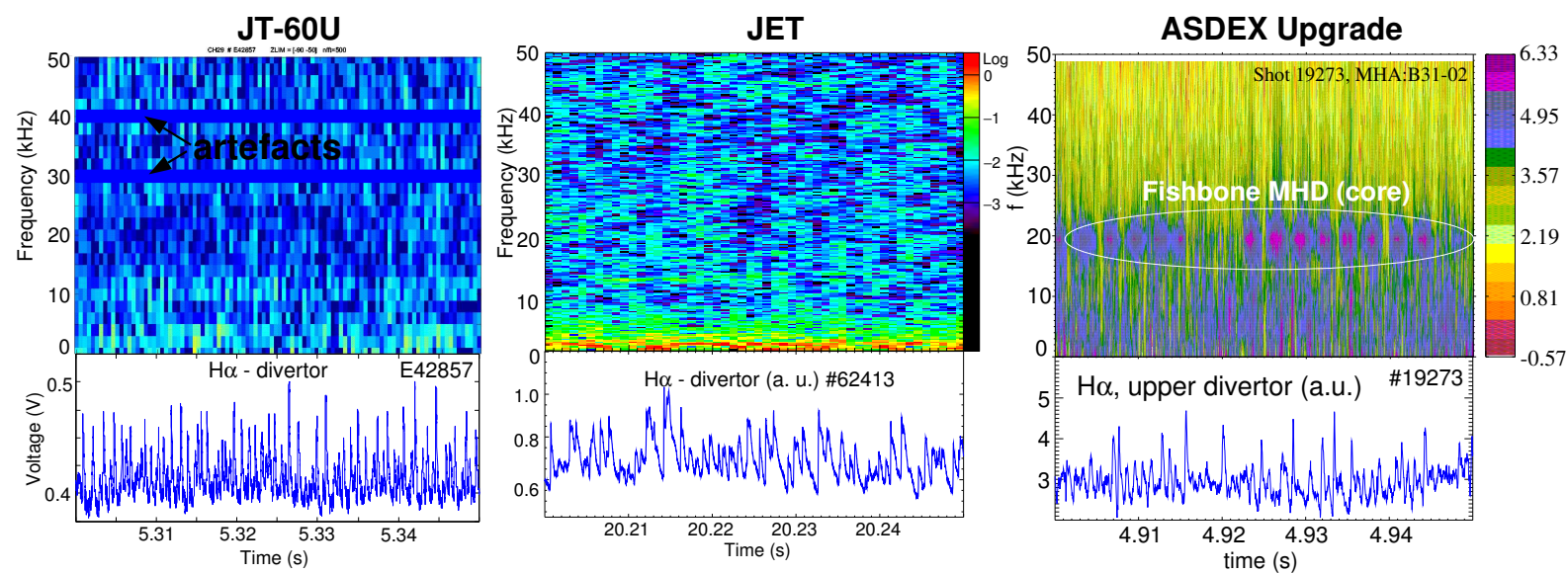

Figure 5: Magnetic spectrograms during grassy ELM phases. JT-60U pulse E42857: $I_{p}=1.0 \mathrm{MA}$, $B_{t}=3.6 \mathrm{~T}, q_{95}=6.4, P_{\text {aux }} \approx 20 \mathrm{MW}, \beta_{\text {pol }}=2.0$, JET pulse 62413: $I_{p}=1.2 \mathrm{MA}, B_{t}=2.7 \mathrm{~T}, q_{95}=6.9$, $P_{\text {aux }} \approx 23 \mathrm{MW}, \beta_{\text {pol }}=1.8$, ASDEX Upgrade pulse 19273: $I_{p}=0.8 \mathrm{MA}, B_{t}=3 \mathrm{~T}, q_{95}=6.2, P_{\text {aux }} \approx 18 \mathrm{MW}$, $\beta_{\text {pol }}=1.8$

\section{2. high $\beta_{p o l}$ grassy ELM experiments at ASDEX Upgrade}

The high $\beta_{\text {pol }}$ strategy has also been tried recently on ASDEX Upgrade. Pure grassy ELMs were found with a configuration close to DN $[23,8]$. The plasma shape is close to the original type-II shape [9], with stronger heating (14 MW) similar to the improved H-modes which can be integrated with type-II ELMs [19]. In contrast to these earlier experiments $q_{95}$ is increased to $\approx 7$ to access the grassy ELM regime according to the operational boundaries determined at JT-60U. This is done by increasing $B_{t}$ to $3 \mathrm{~T}$ keeping $I_{p}=0.8 \mathrm{MA}$. The resulting grassy ELM regime could indeed be accessed at lower densities as compared to the original type-II regime. For a very similar SN configuration only mixed type-I/grassy phases were obtained. The spectrograms of the magnetic pick-up probes are similar to what has been found on JT$60 \mathrm{U}$ and JET, although the affected frequency region extends up to $10 \mathrm{kHz}$, perhaps due to the smaller machine size. It is not clear in how far these high- $q_{95}$ grassy ELMs are different from the earlier type-II ELMs, in as much as there are differences in the MHD spectra. As already mentioned, type-II ELMs are also found in improved H-modes [19]. These plasmas have $q_{95}=3.5$ lower than the earlier type-II plasmas but a $\beta_{\text {pol }} \approx 1.6$ close to that of the grassy ELMs at high $q_{95}$. They do not show enhanced fluctuations below $5 \mathrm{kHz}$ nor the modes at a few $10 \mathrm{kHz}$.

\section{Conclusions}

H-modes with an edge behaviour reminiscent of EDA, high density type-II ELMs and high $\beta_{p o l}$ grassy ELMs have been developed at JET, of which only the latter was fully controlled, whereas the others were limited by radiation collapses due to central density peaking. The operational regime is restricted to plasma currents below $1 \mathrm{MA}$ for the pure type-II ELMs. The mixed type-I/II phases observed at JET earlier at 2.5 MA, could also not be transfered to pure type-II phases by using quasi-DN configurations nor higher values of $q_{95}$. In dimensionless parameters, essentially $\rho^{*}$ and $v^{*}$ decrease with increasing current. Although the change in $\rho^{*}$ is larger, the change in $v^{*}$ is usually favoured as an explanation for this current scaling, since it directly influences the edge bootstrap current and therefore the stability of edge current driven peeling modes $[24,25]$. The plasmas which were meant to be dimensionlessly identical to either the EDA mode in Alcator C-mod or to the type-II ELMs of ASDEX Upgrade were very similar 
in terms of mode activity observed in the magnetic and reflectometer signals in JET, indicating a close relation between both phenomena. Nevertheless it must be noted that only part of this turbulence footprint matches the observations on Alcator C-mod and ASDEX Upgrade. A striking difference between both sets of experiments is that the EDA experiments were done in the same SN configuration which shows the mixed type-I/II ELMs at higher current whereas the type-II identity shots were done in a QDN configuration. This either indicates that the closeness to QDN is not important or that a quantity related to QDN, such as magnetic shear in the steep gradient region, is generated by other moments of the shape in this specific SN configuration. The experience from ASDEX Upgrade clearly favours the latter explanation. Also the effect of QDN on the high $\beta_{p o l}$ plasmas in ASDEX Upgrade and possibly also on JET indicate that it can be an important parameter. For the high $\beta_{p o l}$ scheme, the current is limited to 1.2 MA, but this is due to a limit in additional heating power. The high $\beta_{\text {pol }}$ discharges on JET and ASDEX Upgrade showed similar fluctuations in the MHD spectrogram as observed in JT-60U. Nevertheless, they were a factor 4 higher in $v^{*}$ than those observed in JT-60U $(0.4$ vs. 0.1), whereas in JET the low $l_{i}$ discharges with high $\beta_{p o l}$ reached approximately the JT-60U values but did not show grassy ELMs. This is especially striking since the JT-60U discharges are usually also operated with early heating. An experimental separation of the relevance of $v^{*}, l_{i}, q_{95}$ and closeness to DN for the appearance of grassy ELMs at high $\beta_{p o l}$ in JET is still outstanding.

In view of a future reactor plasma, our results suggest that only the high $\beta_{\text {pol }}$ scheme may be extrapolatable, but means have to be found to run it at lower values of $q_{95}$, as was possible on JT-60U using very strong shaping $(\delta \approx 0.6)[20]$.

\section{References}

[1] LOARTE, A. et al., J. Nucl. Mater. 290-293 (2001) 1013.

[2] SUTTROP, W. et al., pages IAEA-CN-116/EX/1-4, this conference.

[3] SAIBENE, G. et al., Plasma Phys. Controlled Fusion 44 (2002) 1769.

[4] SARTORI, R. et al., pages IAEA-CN-116/EX/6-3, this conference.

[5] PEREZ, C. P. et al., Plasma Phys. Controlled Fusion 46 (2004) 61.

[6] SMEULDERS, P. et al., Plasma Phys. Controlled Fusion 41 (1999) 1303.

[7] SAIBENE, G. et al., Plasma Phys. Controlled Fusion, to be submitted.

[8] STOBER, J. et al., Nucl. Fusion, extended version of this conference contribution, to be submitted.

[9] STOBER, J. et al., Nucl. Fusion 41 (2001) 1123.

[10] SUTTROP, W. et al., Fusion Science and Technology 44 (2003) 636.

[11] SUTTROP, W. A. et al., in Proc. of the 19th IAEA Conference Fusion Energy, Lyon, 2002, volume IAEA-CSP-19/CD, pages IAEA-CN-94/EX/P5-07, Vienna, 2003, IAEA.

[12] MOSSESSIAN, D. A. et al., Plasma Phys. 10 (2003) 1720.

[13] MADDISON, G. P. et al., in ECA (CD-ROM, Proc. of the 30th EPS Conference on Controlled Fusion and Plasma Physics, St. Petersburg, 2003), volume 27A, P-1.109, Geneva, 2003, EPS.

[14] MOSSESSIAN, D. A. et al., in ECA (CD-ROM, Proc. of the 30th EPS Conference on Controlled Fusion and Plasma Physics, St. Petersburg, 2003), volume 27A, P-3.182, Geneva, 2003, EPS.

[15] MAHDAVI, M. A. et al., Nucl. Fusion 42 (2002) 52.

[16] STOBER, J. et al., Nucl. Fusion 43 (2003) 1265.

[17] RICE, J. E. et al., Nucl. Fusion 43 (2003) 781.

[18] VALOVIC, M. et al., Plasma Phys. Controlled Fusion 44 (2002) 1911.

[19] SIPS, A. C. C. et al., Plasma Phys. Controlled Fusion 44 (2002) A151.

[20] KAMADA, Y. et al., Plasma Phys. Controlled Fusion 44 (2002) A279.

[21] KAMADA, Y. et al., Plasma Phys. Controlled Fusion 42 (2000) A247.

[22] SAARELMA, S. et al., Plasma Phys. Controlled Fusion 46 (2004) 1259.

[23] HORTON, L. D. et al., 2004, Plasma Phys. Controlled Fusion, EPS 2004 special issue.

[24] SAARELMA, S. et al., Nucl. Fusion 43 (2003) 262.

[25] LÖNNROTH, J.-S. et al., Plasma Phys. Controlled Fusion 46 (2004) 767. 